\title{
THE SOLUTION OF LINEAR INTEGRAL EQUATIONS BY MEANS OF WIENER INTEGRALS
}

\author{
THEODORE G. OSTROM
}

1. Introduction. In this paper, we obtain expressions for the solution, resolvent kernel, and Fredholm determinant of the integral equation

$$
z(t)=x(t)+\int_{0}^{1} K(t, s) x(s) d s
$$

in terms of Wiener integrals. Besides appealing to the general interest which is always inherent in the relating of two apparently diverse fields, these results are of possible importance in two ways: (a) Though the Wiener integrals involved can be evaluated at present only when the kernel takes on certain relatively simple forms, it may be possible in the future to obtain at least approximate evaluations which will in turn offer approximate solutions of the integral equation which may converge faster than the Fredholm solution. Here we might mention specifically the case where the integral equation contains a parameter of large absolute value; (b) As a means of evaluating Wiener integrals in terms of the known (Fredholm) solution of the integral equation.

The Wiener integral is based on a measure defined by Wiener [1 ${ }^{1}$ on the space $C$ of all real functions $x(t)$ continuous on the interval $0 \leqq t \leqq 1$ and vanishing at $t=0$. Cameron and Martin have investigated its properties and have, in particular, discovered how it transforms under translations [2] and linear transformations [3]. They have also been able to express the solution of a class of nonlinear integral equations in terms of limits of Wiener integrals [4]. We shall obtain our results by using their theorems on translations and linear transformations.

2. The basic solution. Given the integral equation

$$
z(t)=F[x \mid t],
$$

where

$$
F[x \mid t]=x(t)+\int_{0}^{1} K(t, s) x(s) d s,
$$

Presented to the Society, April 26, 1947; received by the editors February 5, 1948, and, in revised form, April 5, 1948.

${ }^{1}$ Numbers in brackets refer to the references cited at the end of the paper. 
suppose that the following conditions are satisfied:

1. $z(t) \in C$ and $z^{\prime}(t)$ exists and is of bounded variation on the interval $(0,1)$.

2.

$$
K(t, s)=\left\{\begin{array}{lr}
K_{1}(t, s), & 0 \leqq t<s, \\
K_{2}(t, s), & s<t \leqq 1 \\
\left(K_{1}+K_{2}\right) / 2, & t=s,
\end{array}\right.
$$

where $K_{1}(t, s)$ is continuous, $\{0 \leqq t \leqq s, 0 \leqq s \leqq 1\} ; K(0, s)=0,0 \leqq s \leqq 1$, and $K_{2}(t, s)$ is continuous, $\{0 \leqq s \leqq t, 0 \leqq t \leqq 1\}$.

3. $K(t, s)$ is absolutely continuous in $t$ for almost all $s, 0 \leqq s \leqq 1$, after the jump at $t=s$ is removed by the addition of a step function.

4. For almost all $s, \partial K(t, s) / \partial t$ is essentially of bounded variationthat is, there exists a measurable function $K_{t}^{*}(t, s)$ which is of bounded variation in $t$ for each $s$ and which for almost all $(t, s)\{0 \leqq t \leqq 1$, $0 \leqq s \leqq 1\}$ is equal to $\partial K(t, s) / \partial t$.

5. $K_{t}^{*}(t, s)$ can be so chosen that

$$
\begin{aligned}
& \int_{0}^{1} \sup _{0 \leqq t \leqq 1}\left|K_{t}^{*}(t, s)\right| d s<\infty, \\
& \int_{0}^{1} \operatorname{var}_{0 \leqq t \leqq 1}\left[K_{t}^{*}(t, s)\right] d s<\infty .
\end{aligned}
$$

6. $J(s)=K_{2}(s, s)-K_{1}(s, s)$ is of bounded variation.

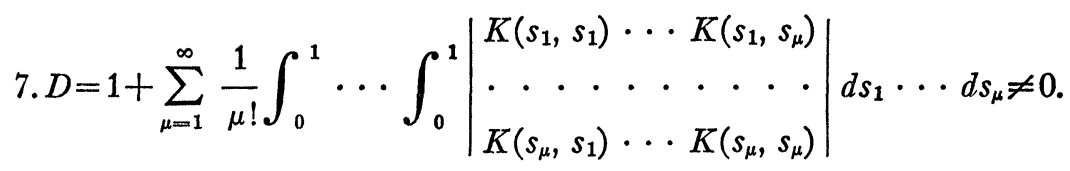

We shall show in this section that under the above conditions our integral equation can be solved in terms of Wiener integrals. The explicit form of the solution is given in the following theorem:

THEOREM I. Let $z(t)$ and $K(t, s)$ satisfy conditions 1-7 above. Then the solution of the equation (2.1) with $F[x \mid t]$ defined by (2.2) is given by the formula:

$$
\begin{aligned}
x(t)=|D| \exp & \left(-\int_{0}^{1}\left[z^{\prime}(s)\right]^{2} d s\right) \\
& \cdot \int_{c}^{w} u(t) \exp \left(2 \int_{0}^{1} z^{\prime}(s) d F[u \mid s]-\Phi(u)\right) d_{w} u
\end{aligned}
$$

where 


$$
\begin{aligned}
\Phi(u)= & \int_{0}^{1}\left[\frac{d}{d \xi} \int_{0}^{1} K(\xi, s) u(s) d s\right]^{2} d \xi \\
& +2 \int_{0}^{1}\left[\int_{0}^{1} \frac{\partial K(\xi, s)}{\partial \xi} u(s) d s\right] d u(\xi)+\int_{0}^{1} J(\xi) d\left[u^{2}(\xi)\right] .
\end{aligned}
$$

Proof. ${ }^{2}$ Under the conditions of Theorem I, the equation (2.1) determines a 1-1 mapping of $C$ into itself. Hence for each $z(t)$ in $C$ there is a unique solution $x(t)$ belonging to $C$ which can be written as

$$
x(t)=G[z \mid t]
$$

Thus

$$
G[F(x \mid \cdot) \mid t] \equiv x(t) .
$$

Moreover, it is apparent that, if, in (2.1) and (2.2), $G[y \mid t]$ is the corresponding solution when $z(t)$ is replaced by $y(t)$, then $a G[z \mid t]$ $+b G[y \mid t]$ is the solution when $z(t)$ is replaced by $a z(t)+b y(t)$, where $a$ and $b$ are constants. That is, $G[a z+b y \mid t]=a G[z \mid t]+b G[y \mid t]$. Then, since $G$ is odd,

$$
\int_{c}^{w} G[y \mid t] d_{w} y=0
$$

and since $\int_{c}^{w} d_{w} y=1$, we have

$$
\int_{c}^{w} G[z+y \mid t] d_{w} y=G[z \mid t] \text {. }
$$

Next we quote a theorem of Cameron and Martin [2]:

Let $x_{0}(t)$ be a given continuous function vanishing at $t=0$ and having $a$ derivative $x_{0}^{\prime}(t)$ of bounded variation, $0 \leqq t \leqq 1$. Then if $H[y]$ is any functional for which either member of (2.8) (below) exists, the other member also exists and the equation (2.8) holds:

$$
\begin{aligned}
\int_{c}^{w} H[y] d_{w} y & =\exp \left(-\int_{0}^{1}\left[x_{0}^{\prime}(s)\right]^{2} d s\right) \\
& \cdot \int_{c}^{w} H\left[x+x_{0}\right] \exp \left(-2 \int_{0}^{1} x_{0}^{\prime}(s) d x(s)\right) d_{w} x
\end{aligned}
$$

In (2.8), let $H[y]=G[z+y]$ and let $x_{0}=-z$, noting that, from (2.7), we know that $\int_{c}^{w} G[z+y] d_{w} y$ exists and by condition 1 of the hy-

\footnotetext{
${ }^{2}$ At the suggestion of the referee, the form of the first part of this argument has been modified slightly, so that reference to the Fredholm resolvent kernel could be postponed to a later stage of the paper.
} 
potheses, $Z(t)$ satisfies the conditions placed on $x_{0}(t)$. (2.8) becomes:

$$
\begin{aligned}
\int_{c}^{w} G[z+y] d w y & \\
& =\exp \left(-\int_{0}^{1}\left[z^{\prime}(s)\right]^{2} d s\right) \int_{c}^{w} G[x] \exp \left(2 \int_{0}^{1} z^{\prime}(s) d x(s)\right) d_{w} x .
\end{aligned}
$$

Applying (2.7) and changing the variable of integration on the right to $y$ so that confusion with $x(t)$ in (2.2) may be avoided, we get

$$
\begin{aligned}
G[z \mid t]= & \int_{c}^{w} G[(z+y) \mid t] d_{w} y \\
= & \exp \left(-\int_{0}^{1}\left[z^{\prime}(s)\right]^{2} d s\right) \int_{c}^{w} G[y \mid t] \\
& \cdot \exp \left(2 \int_{0}^{1} z^{\prime}(s) d y(s)\right) d_{w} y .
\end{aligned}
$$

Next we shall consider the transformation

$$
y(t)=u(t)+\int_{0}^{1} K(t, s) u(s) d s \equiv F[u \mid t] .
$$

By another theorem of Cameron and Martin [3], if $K$ satisfies hypotheses $2-7$, and if $H[y]$ is any measurable functional for which either member of (2.10) (below) exists, then the other member exists and the equality holds:

$$
\begin{array}{r}
\int_{c}^{w} H[y] d_{w} y=|D| \int_{c}^{w} H\left[u+\int_{0}^{1} K(\cdot, s) u(s) d s\right] \\
\cdot \exp (-\Phi(u)) d_{w} u,
\end{array}
$$

where $\Phi(u)$ is defined in (2.4).

Now in (2.10), let $H[y]=G[y] \exp \left(2 \int_{0}^{1} z^{\prime}(s) d y(s)\right)$ since from (2.9) we know that $\int_{c}^{w} G[y] \exp \left(2 \int_{0}^{1} z^{\prime}(s) d y(s)\right) d_{w} y$ exists and since Wiener integrability implies measurability, this value of $H[y]$ is a measurable functional. We obtain

$$
\begin{aligned}
\int_{c}^{w} G[y] & \exp \left(2 \int_{0}^{1} z^{\prime}(s) d y(s)\right) d_{w} y \\
= & |D| \int_{c}^{w} G[F(u \mid \cdot)] \exp \left(2 \int_{0}^{1} z^{\prime}(s) d F[u \mid s]-\Phi(u)\right) d_{w} u,
\end{aligned}
$$

which reduces, on applying (2.6), to 


$$
\begin{aligned}
& \int_{c}^{w} G[y \mid t] \exp \left(2 \int_{0}^{1} z^{\prime}(s) d y(s)\right) d_{w} y \\
& =|D| \int_{c}^{w} u(t) \exp \left(2 \int_{0}^{1} z^{\prime}(s) d F[u \mid s]-\Phi(u)\right) d_{w} u .
\end{aligned}
$$

Putting together (2.9) and (2.11), we get

$$
\begin{array}{r}
G[z \mid t]=|D| \exp \left(-\int_{0}^{1}\left[z^{\prime}(s)\right]^{2} d s\right) \int_{c}^{w} u(t) \\
\cdot \exp \left(2 \int_{0}^{1} z^{\prime}(s) d F[u \mid s]-\Phi(u)\right) d_{w} u .
\end{array}
$$

But, by (2.5), $x(t)=G[z \mid t]$ is the solution of (2.1) and our theorem is proved.

3. Reduction to linear form. This solution can be put into a form in which the Wiener integrals are simpler, although the solution is a more complicated function of the integrals. The process, particularly as regards justification of some of the steps, is somewhat laborious hence we shall only summarize the argument.

If, in (2.1), $z(t)$ be replaced by $\lambda z(t)$, the solution $x(t)$ will be replaced by $\lambda x(t)$. Hence

$$
\begin{aligned}
\lambda x(t)=|D| & \exp \left(-\lambda^{2} \int_{0}^{1}\left[z^{\prime}(s)\right]^{2} d s\right) \int_{0}^{w} u(t) \\
& \cdot \exp \left(2 \lambda \int_{0}^{1} z^{\prime}(s) d F[u \mid s]-\Phi(u)\right) d_{w} u .
\end{aligned}
$$

It can be shown that differentiation under the Wiener integral sign is justifiable provided that $z^{\prime \prime}(s)$ is continuous. If we assume $z^{\prime \prime}(s)$ continuous, and differentiate both sides of (3.1) with respect to $\lambda$,

$$
\begin{aligned}
x(t)=|D| \exp & \left(-\lambda^{2} \int_{0}^{1}\left[z^{\prime}(s)\right]^{2} d s\right)\left\{-2 \lambda \int_{0}^{1}\left[z^{\prime}(s)\right]^{2} d s\right\} \\
& \cdot \int_{c}^{w} u(t) \exp \left(2 \lambda \int_{0}^{1} z^{\prime}(s) d F[u \mid s]-\Phi(u)\right) d_{w} u
\end{aligned}
$$

2)

$$
\begin{gathered}
+|D| \exp \left(-\lambda^{2} \int_{0}^{1}\left[z^{\prime}(s)\right]^{2} d s\right) \int_{0}^{w} u(t) \\
\cdot \exp \left(2 \lambda \int_{0}^{1} z^{\prime}(s) d F[u \mid s]-\Phi(u)\right)\left[2 \int_{0}^{1} z^{\prime}(s) d F(u \mid s)\right] d_{w} u .
\end{gathered}
$$


Now (3.2) is an identity in $\lambda$ which holds in particular when $\lambda=0$, hence

$$
\text { (3.3) } x(t)=2|D| \int_{c}^{w} u(t)\left[\int_{0}^{1} z^{\prime}(s) d F(u \mid s)\right] \exp (-\Phi(u)) d_{w} u .
$$

Now $F[u \mid s]=u(s)+\int_{0}^{1} K(s, \xi) u(\xi) d \xi$, so that we obtain by substitution in (3.3)

$$
\begin{array}{r}
x(t)=2|D| \int_{c}^{w} u(t)\left[\int_{0}^{1} z^{\prime}(s) d u(s)\right] \exp (-\Phi(u)) d_{w} u \\
+2|D| \int_{c}^{w} u(t)\left\{\int_{0}^{1} z^{\prime}(s) \frac{d}{d s}\left[\int_{0}^{1} K(s, \xi) u(\xi) d \xi\right] d s\right\} \\
\cdot \exp (-\Phi(u)) d_{w} u .
\end{array}
$$

Substituting $\int_{0}^{1} z^{\prime}(s) d u(s)=z^{\prime}(1) u(1)-\int_{0}^{1} u(s) d\left[z^{\prime}(s)\right]$ and

$$
d \int_{0}^{1} K(s, \xi) u(\xi) d \xi / d s=\int_{0}^{1} \partial K(s, \xi) / \partial s u(\xi) d \xi+J(s) u(s)
$$

we get

$$
\begin{aligned}
x(t)= & 2|D| \int_{c}^{w} u(t)\left\{u(1) z^{\prime}(1)-\int_{0}^{1} u(s) d z^{\prime}(s)\right\} \\
& +2|D| \int_{c}^{w} u(t)\left\{\int_{0}^{1} z^{\prime}(s)\left[\int_{0}^{1} \frac{\partial K(s, \xi)}{\partial s} u(\xi) d \xi\right] d s\right\} \\
& +2|D| \int_{c}^{w} u(t)\left\{\int_{0}^{1} z^{\prime}(s) J(s) u(s) d s\right\} \exp (-\Phi(u)) d_{w} u \\
& \exp (-\Phi(u)) d_{w} u .
\end{aligned}
$$

Interchanging the order of Wiener integration and ordinary integration (which can be justified by the mixed Fubini theorem), we obtain

$$
\begin{aligned}
x(t)= & 2|D|\left\{z^{\prime}(1) \int_{c}^{w} u(t) u(1) \exp (-\Phi) d_{w} u\right. \\
& -\int_{0}^{1} \int_{c}^{w} u(t) u(s) \exp (-\Phi) d_{w} u d z^{\prime}(s) \\
& +\int_{0}^{1} z^{\prime}(s)\left[\int_{0}^{1} \frac{\partial K(s, \xi)}{\partial s} \int_{c}^{w} u(t) u(\xi) \exp (-\Phi) d_{w} u d \xi\right] d s \\
& \left.+\int_{0}^{1} z^{\prime}(s) J(s)\left[\int_{c}^{w} u(t) u(s) \exp (-\Phi) d_{w} u\right] d s\right\}
\end{aligned}
$$




$$
\begin{aligned}
& =2|D|\left\{z^{\prime}(1) W(t, 1)-\int_{0}^{1} W(t, s) d\left[z^{\prime}(s)\right]\right. \\
& +\int_{0}^{1} z^{\prime}(s)\left[\int_{0}^{1} \frac{\partial K(s, \xi)}{\partial s} W(t, \xi) d \xi\right] d s \\
& \left.\quad+\int_{0}^{1} z^{\prime}(s) J(s) W(t, s) d s\right\},
\end{aligned}
$$

where $W(t, s)=\int_{c}^{w} u(t) u(s) / \exp (-\Phi(u)) d_{w} u$ and $z^{\prime \prime}(s)$ is continuous. It will be shown later that $W(t, s)$ is continuous in $s$, hence this may be written ( since $W(t, 0)=0$ )

$$
\begin{aligned}
x(t)=2 \mid & D \mid\left\{\int_{0}^{1} z^{\prime}(s) d_{s}[W(t, s)]\right. \\
& +\int_{0}^{1} z^{\prime}(s)\left[\int_{0}^{1} \frac{\partial K(s, \xi)}{\partial s} W(t, \xi) d \xi\right] d s \\
& \left.+\int_{0}^{1} z^{\prime}(s) J(s) W(t, s) d s\right\} .
\end{aligned}
$$

4. Expression of $D$ and the Fredholm resolvent kernel in terms of Wiener integrals.

THEOREM II. If, in addition to satisfying the conditions of Theorem I, $K_{1}(t, s)$ and $K_{2}(t, s)$ have continuous partial second derivatives with respect to $t$, then

$$
R(t, s)=-2\left\{\int_{c}^{w} \exp (-\Phi(u)) d_{w} u\right\}^{-1}\left\{\frac{\partial^{2} W(t, s)}{\partial s^{2}}\right.
$$

for all $s, t$ except $s=t$, where

$$
\left.+\frac{\partial^{2}}{\partial s^{2}} \int_{0}^{1} K(s, \xi) W(t, \xi) d \xi\right\}
$$

$$
W(t, s)=\int_{c}^{w} u(t) u(s) \exp (-\Phi(u)) d_{w} u
$$

and $R(t, s)$ is the resolvent kernel

Proof. Equation (3.6) holds if $z^{\prime \prime}(s)$ is continuous; let us restrict $z(s)$ still further, so that $z^{\prime}(0)=0$. Then

$$
z^{\prime}(1)=\int_{0}^{1} z^{\prime \prime}(s) d s .
$$

Moreover, we can write 


$$
\begin{gathered}
\int_{0}^{1} W(t, s) d z^{\prime}(s)=\int_{0}^{1} W(t, s) z^{\prime \prime}(s) d s \\
\int_{0}^{1} z^{\prime}(s) \frac{\partial K(s, \xi)}{\partial s} d s=\int_{0}^{\xi} z^{\prime}(s) \frac{\partial K_{1}(s, \xi)}{\partial s} d s+\int_{\xi}^{1} z^{\prime}(s) \frac{\partial K_{2}(s, \xi)}{\partial s} d s \\
=K(1, \xi) z^{\prime}(1)-\int_{0}^{1} K(s, \xi) z^{\prime \prime}(s) d s-J(\xi) z^{\prime}(\xi)
\end{gathered}
$$

(integrating each integral by parts and then recombining into a single integral). Hence

$$
\begin{aligned}
\int_{0}^{1} z^{\prime}(s) & {\left[\int_{0}^{1} \frac{\partial K(s, \xi)}{\partial s} W(t, \xi) d \xi\right] d s } \\
= & {\left[\int_{0}^{1} K(1, \xi) W(t, \xi) d \xi\right] \int_{0}^{1} z^{\prime \prime}(s) d s } \\
& -\int_{0}^{1} z^{\prime \prime}(s)\left[\int_{0}^{1} K(s, \xi) W(t, \xi) d \xi\right] d s-\int_{0}^{1} W^{\prime}(t, \xi) J(\xi) z^{\prime}(\xi) d \xi .
\end{aligned}
$$

Thus, (3.6) can be written

$$
\begin{aligned}
x(t)= & 2|D| \int_{0}^{1} z^{\prime \prime}(s)\{W(t, 1)-W(t, s) \\
& \left.+\int_{0}^{1}[K(1, \xi)-K(s, \xi)] W(t, \xi) d \xi\right\} d s .
\end{aligned}
$$

But also, under the conditions of Theorem I, we have, by ordinary Fredholm theory,

$$
x(t)=z(t)+\int_{0}^{1} R(t, s) z(s) d s .
$$

Now $z(t)=\int_{0}^{1} z^{\prime}(s) \gamma(t, s) d s$, where

$$
\gamma(t, s)= \begin{cases}1, & 0 \leqq s \leqq t, \\ 0, & t<s \leqq 1 .\end{cases}
$$

Integrating by parts and noting that the indefinite integral of $\gamma(t, s)$ with respect to $s$ is

$$
\min (t, s)= \begin{cases}t, & t \leqq s, \\ s, & t \geqq s,\end{cases}
$$

we obtain $z(t)=t \int_{0}^{1} z^{\prime \prime}(s) d s-\int_{0}^{1} z^{\prime \prime}(s) \min (t, s) d s$. Finally, let 


$$
R_{1}(t, s)=\int_{0}^{s} R(t, \xi) d \xi, \quad R_{2}(t, s)=\int_{0}^{0} R_{1}(t, \xi) d \xi ;
$$

then $\int_{0}^{1} R(t, s) z(s) d s=R_{1}(t, 1) z(1)-\int_{0}^{1} R_{1}(t, s) z^{\prime}(s) d s$. Integrating by parts again, and replacing $z(1)$ by

$$
\int_{0}^{1} z^{\prime \prime}(s) d s-\int_{0}^{1} s z^{\prime \prime}(s) d s
$$

we get

$$
\begin{aligned}
\int_{0}^{1} R(t, s) z(s) d s= & R_{1}(t, 1)\left[\int_{0}^{1} z^{\prime \prime}(s) d s-\int_{0}^{1} s z^{\prime \prime}(s) d s\right] \\
& -R_{2}(t, 1) \int_{0}^{1} z^{\prime \prime}(s) d s+\int_{0}^{1} R_{2}(t, s) z^{\prime \prime}(s) d s
\end{aligned}
$$

and

$$
\begin{aligned}
x(t)=\int_{0}^{1} z^{\prime \prime}(s)\left\{t-\min (t, s)+R_{1}(t, 1)(1-s)\right. & \\
& \left.-R_{2}(t, 1)+R_{2}(t, s)\right\} d s
\end{aligned}
$$

which gives us, when combined with (4.2),

$$
\begin{aligned}
2|D| & \int_{0}^{1} z^{\prime \prime}(s)\{W(t, 1)-W(t, s) \\
& +\int_{0}^{1}[K(1, \xi)-K(s, \xi) W(t, \xi) d \xi\} d s \\
= & \int_{0}^{1} z^{\prime \prime}(s)\left\{t-\min (t, s)+R_{1}(t, 1)(1-s)\right. \\
& \left.\quad-R_{2}(t, 1)+R_{2}(t, s)\right\} d s .
\end{aligned}
$$

Transposing, we get

$$
\begin{gathered}
\int_{0}^{1} z^{\prime \prime}(s)\{2|D| W(t, 1)-2|D| W(t, s) \\
+(4.5) \quad+2|D| \int_{0}^{1}[K(1, \xi)-K(s, \xi)] W(t, \xi) d \xi \\
\left.-t+\min (t, s)-R_{1}(t, 1)(1-s)+R_{2}(t, 1)-R_{2}(t, s)\right\} d s=0 .
\end{gathered}
$$

Now (4.5) holds for $z(s)$ any function satisfying the conditions: 
(a) $z(0)=z^{\prime}(0)=0$,

(b) $z(t)$ continuous, $0 \leqq t \leqq 1$,

(c) $z^{\prime}(t)$ is of bounded variation on $(0,1)$,

(d) $z^{\prime \prime}(t)$ continuous on $(0,1)$.

Condition (d) implies (b) and (c); moreover, given any function $z^{\prime \prime}(t)$, the constants of integration can be so determined that (a) will be satisfied. Hence $z^{\prime \prime}(s)$ can be any continuous function. Next let us write (4.5) in the form

$$
\int_{0}^{1} z^{\prime \prime}(s) \phi(s) d s=0,
$$

that is, $\phi(s)$ represents the expression in brackets in (4.5). We see that $\phi(s)$ will be continuous if $W(t, s)$ is a continuous function of $s$. But, by (2.10), (2.2), (2.5) and (2.6),

$$
\begin{aligned}
W(t, s)= & \int_{c}^{w} u(t) u(s) \exp (-\Phi) d_{w} u=\frac{1}{|D|} \int_{c}^{w} G[v \mid t] G[v \mid s] d_{w} v \\
= & \frac{1}{|D|} \int_{c}^{w}\left[v(t)+\int_{0}^{1} R(t, \xi) v(\xi) d \xi\right] \\
& \cdot\left[v(s)+\int_{0}^{1} R(s, \xi) v(\xi) d \xi\right] d_{w} v .^{3}
\end{aligned}
$$

Multiplying out and interchanging order of integration, we get

$$
\begin{aligned}
W(t, s)= & \frac{1}{|D|}\left\{\int_{c}^{w} v(t) v(s) d_{w} v+\int_{0}^{1} R(s, \xi) \int_{c}^{w} v(t) v(\xi) d_{w} v d \xi\right. \\
& +\int_{0}^{1} R(t, \xi) \int_{c}^{w} v(s) v(\xi) d_{w} v d \xi \\
& +\int_{0}^{1} \int_{0}^{1} R(t, \xi) R(s, \eta) \int_{c}^{w} v(\xi) v(\eta) d_{w} v d \xi d \eta \\
= & \frac{1}{2|D|}\left\{\min (t, s)+\int_{0}^{1} R(s, \xi) \min (t, \xi) d \xi\right. \\
& +\int_{0}^{1} R(t, \xi) \min (s, \xi) d \xi \\
& \left.+\int_{0}^{1} \int_{0}^{1} R(t, \xi) R(s, \eta) \min (\xi, \eta) d \xi d \eta\right\} .
\end{aligned}
$$

${ }^{3}$ Using here the fact that the solution of $(2.1)$ can be written alternately as $x(t)=G[z \mid t]$ or $x(t)=z(t)+\int_{0}^{1} R(t, s) z(s) d s$, so that these two expressions are equal. 
Since $R(t, s)$ is continuous at any point of continuity of $K(t, s)$, the right-hand side of $(4.7)$ is continuous Thus $W(t, s)$ and, therefore, $\phi(s)$ are continuous on $(0,1)$. Now $z^{\prime \prime}(s)$ may, in particular, be any of the complete orthonormal sets of polynomials on $(0,1)$. From (4.6) and definition of completeness, $\phi(s) \equiv 0$. Therefore

$$
\begin{aligned}
2|D|\{W(t, 1)-W(t, s) & \left.+\int_{0}^{1}[K(1, \xi)-K(s, \xi)] W(t, \xi) d \xi\right\} \\
& -t+\min (t, s)-R_{1}(t, 1)(1-s) \\
& +R_{2}(t, 1)-R_{2}(t, s) \equiv 0
\end{aligned}
$$

Now for every $t \neq s$ we note that each term in (4.8) has a derivative with respect to $s$ except possibly $W(t, s)$; hence $\partial W(t, s) / \partial s$ exists. Differentiating, we get

$$
\begin{aligned}
2|D|\left\{\frac{\partial W(t, s)}{\partial s}+\frac{\partial}{\partial s} \int_{0}^{1} K(s, \xi) W(t, \xi) d \xi\right\} & +\gamma(t, s) \\
& +R_{1}(t, 1)-R_{1}(t, s) \equiv 0 .
\end{aligned}
$$

Differentiating again, we get

$$
-2|D|\left\{\frac{\partial^{2} W(t, s)}{\partial s^{2}}+\frac{\partial^{2}}{\partial s^{2}} \int_{0}^{1} K(s, \xi) W(t, \xi) d \xi\right\}-R(t, s)=0
$$

As in the previous step, we infer the existence of $\partial^{2} W(t, s) / \partial s^{2}$ from the existence of the derivatives of all other terms in the identity.

Finally, we can express $D$ in terms of Wiener integrals. If, in (2.10), we set $H[y] \equiv 1$, then

$$
1=|D| \int_{0}^{w} \exp (-\Phi(u)) d_{w} u .
$$

Hence

$$
|D|=\left\{\int_{0}^{w} \exp (-\Phi(u)) d_{w} u\right\}^{-1}
$$

Theorem II then follows directly from (4.9).

\section{REFERENCES}

1. N. Wiener, Generalized harmonic analysis, Acta Math. vol. 55 (1930) pp. 117258 , especially pp. 214-234.

2. R. H. Cameron and W. T. Martin, Transformations of Wiener integrals under translations, Ann. of Math. vol. 45 (1944) p. 386. 
3. - Transformations of Wiener integrals under a general class of linear transformations, Trans. Amer. Math. Soc. vol. 58 (1945) p. 184.

4. - An expression for the solution of a class of non-linear integral equations, Amer. J. Math. vol. 46 (1944) p. 281.

5. - Evaluation of various Wiener integrals by use of certain Sturm-Liouville differential equations, Bull. Amer. Math. Soc. vol. 51 (1945) pp. 73-89.

Montana State University 